\title{
The CMS RPC detector performance during LHC Run-II data taking
}

\author{
Mehar Ali Shah ${ }^{* \dagger}$ \\ National center for Physics, Quaid-i-Azam University Islamabad, Pakistan \\ E-mail: mashah@cern.ch
}

The CMS experiment at the CERN Large Hadron Collider (LHC) has a redundant muon system composed by three different detector technologies: Cathode Strip Chambers (CSC, in the endcap regions), Drift Tubes (DT, in the barrel region), and Resistive Plate Chambers (RPC, both in the barrel and endcap). The RPC are designed mainly as a trigger detector but they contribute also to the muon reconstruction. Thus the monitoring and the analysis of the system performance are necessary and essential for the final data quality. The main detector characteristics and the hit efficiency and cluster size are presented in the paper. The stability of the system in the conditions of high instantaneous luminosity and high number of pile up (PU) events are presented in a view of history monitoring and stable trend.

EPS-HEP 2017, European Physical Society conference on High Energy Physics 5-12 July 2017

Venice, Italy

${ }^{*}$ Speaker.
${ }^{\dagger}$ On behalf of CMS Collaboration.




\section{Introduction}

\subsection{CMS Experiment}

The Large Hadron Collider (LHC) [1], the most energetic particle accelerator ever built is a double ring structure that collides beams of protons at a center-of-mass energy of $13 \mathrm{TeV}$. Located at one of the 4 interaction points is the Compact Muon Solenoid (CMS) experiment [2], where three types of gaseous detectors are used to identify and measure muons. In the barrel region (the pseudorapidity $|\eta|<1.2$ ), Drift Tube (DT) chambers are used. In the endcaps, Cathode Strip Chambers (CSC) are deployed which cover the region up to $|\eta|<2.4$. In addition to this, resistive plate chambers (RPC) [3] are installed in both barrel and end-cap regions. These RPCs are operated in the avalanche mode to ensure the expected time resolution of $\approx 2 \mathrm{~ns}$ at rates of the order of 10 $\mathrm{kHz} / \mathrm{cm}^{2}$. To monitor and optimise the performance of RPCs within CMS, data taken during 2016 at $13 \mathrm{TeV}$ have been used.

\subsection{The Resistive Plate Chambers}

The CMS-RPC system is composed of double-gap chambers, each $2 \mathrm{~mm}$ gas gap formed by two parallel bakelite electrodes. Copper readout signal electrodes are placed in between the gas-gaps. In the barrel the muon system is made of four coaxial stations, interleaved with iron yokes. The endcap region consists of three iron disks interlayed with 4 RPC stations. The geometry of the RPC strips is mainly driven by the need to have the trigger adjustable on different $p_{T}$ muons. In the barrel, the strip shapes are rectangular while in the endcaps they are trapezoidal. More details about the CMS muon system might be found in the Technical Design Report [4]. The total number of readout channels of the RPC system is larger than 130,000.

\section{RPC Performance during RUN-II}

\subsection{RPC HV Working Point Calibrations}

A high voltage (HV) scan was performed every year: collision data was recorded at several HV settings during a series of runs to define the optimal operating voltage for each chamber. Details can be found in [5,6] for a full explanation of the HV scan, dependence of efficiency on the $\mathrm{HV}$, including the analysis and methodology. The dependence of the avalanche production on the environmental pressure $\mathrm{P}$, temperature $\mathrm{T}$ and the applied $\mathrm{HV}$ can be summarised in an effective $\mathrm{HV}$ equation (2.1).

$$
H V_{e f f}(P, T)=H V\left(P_{0} / P\right)\left(T / T_{0}\right)
$$

Where $\mathrm{HV}_{\text {eff }}$ is effective high voltage, $\mathrm{HV}$ is applied high voltage, and the reference temperature and pressure are $\mathrm{T}_{0}=293 \mathrm{~K}$ and $\mathrm{P}_{0}=965$ mbar respectively. HV50 is defined as the high voltage at which a chamber reaches $50 \%$ of the efficiency plateau. 




(a)

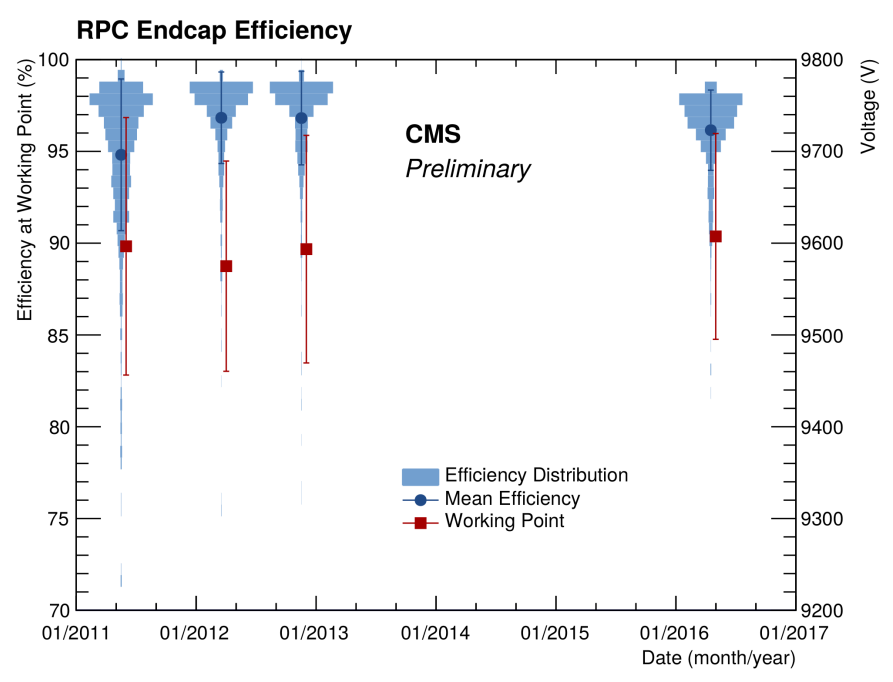

(b)

Figure 1: Efficiency at the working point from the fit evolution and the Voltage at 50\% Efficiency evolution in the Barrel (a) and endcap (b).

The plots shown in figure 1 represent the working point (WP), the efficiency at the WP from the fit evolution and the Voltage at 50\% Efficiency evolution in the Barrel (a) and in the endcap (b). The efficiency at WP distributions have been presented with a light blue color. With a blue, full circle is presented the mean efficiency at WP for each of the HV scans. By red, full squares is represented the mean of the working point distribution for each HV scan with their Standard deviations. In magenta, full triangles represent the mean of the voltage at $50 \%$ efficiency distribution for each HV scan with their standard deviations. The stability during 2011, 2012 and 2016 as shown in figure 1 shows no obvious ageing effects. 


\subsection{RPC Cluster size, Efficiency and Hit Resolution}

Cluster size (CLS) is defined as the number of adjacent strips fired when an avalanche is produced in the RPC. RPC system has stable average cluster size of about 1.8 strips during 2016 as shown in figure 2, which is in agreement with CMS TDR [4]. A little increase in the average CLS in middle of September 2016 is due to applying new higher WP.

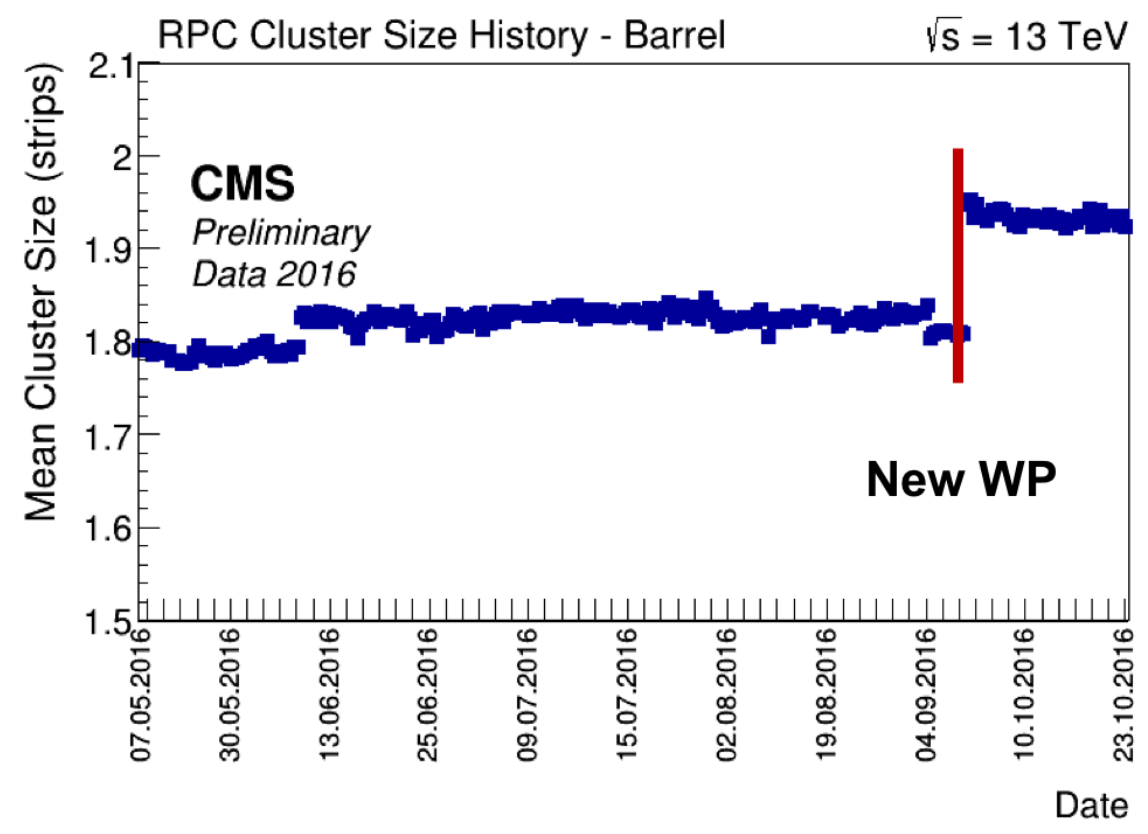

Figure 2: History of the mean Cluster Size for the barrel for 2016 physics data taking.

Residuals for 2 RPC barrel layers are shown in figure 3. There are six layers in total and order of layers corresponds to the distance from the beam pipe, where layer 1 is the closest to it and layer 6 is the outermost one. The residuals are calculated as a difference between the extrapolated point and the center of cluster related to the matched RPC reconstructed hit. The used statistics corresponds to an integrated luminosity of $5.27 \mathrm{fb}^{-1}$ of 2016 proton-proton collisions data. The residual distributions have been fit to Gaussian distributions and the obtained mean and standard deviation are given on the plots. The obtained standard deviations are in agreement with the expectations of less than one strip pitch of the strip for a given layer.

Segment extrapolation method [7] is used to calculate the RPC efficiency. A DT/CSC segment of high quality, associated to a stand-alone muon track, is extrapolated to RPC strip plane. RPC efficiency depends on the atmospheric pressure in the cavern. In order to compensate this dependence, automatic corrections to the HV have been applied during the data taking. A little increase in the average efficiency in middle of September 2016 is due to applying new higher WP. The detailed performance results at previous WP at $13 \mathrm{TeV}$ and their comparison to Run1 data can be found in [8]. Average RPC efficiency during 2016 at $13 \mathrm{TeV}$ was $\approx 95 \%$. During 2016 the RPC system was running with a very stable efficiency. 

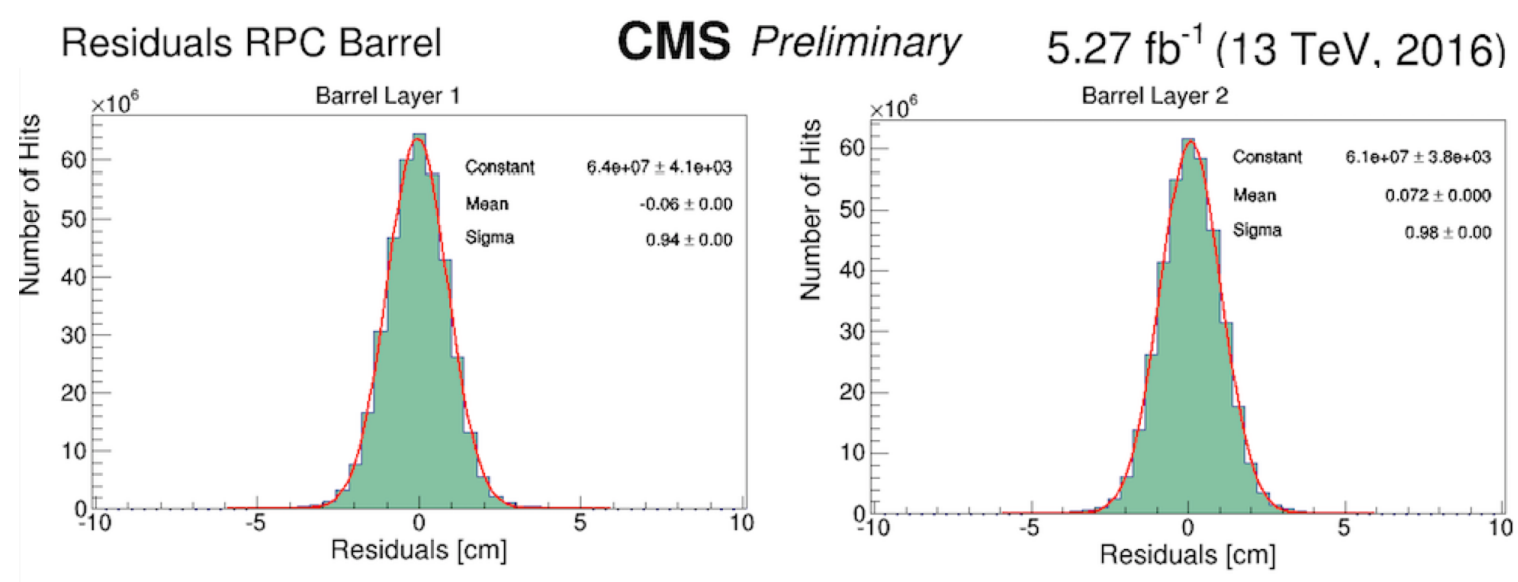

Figure 3: Residuals for 2 barrel layers

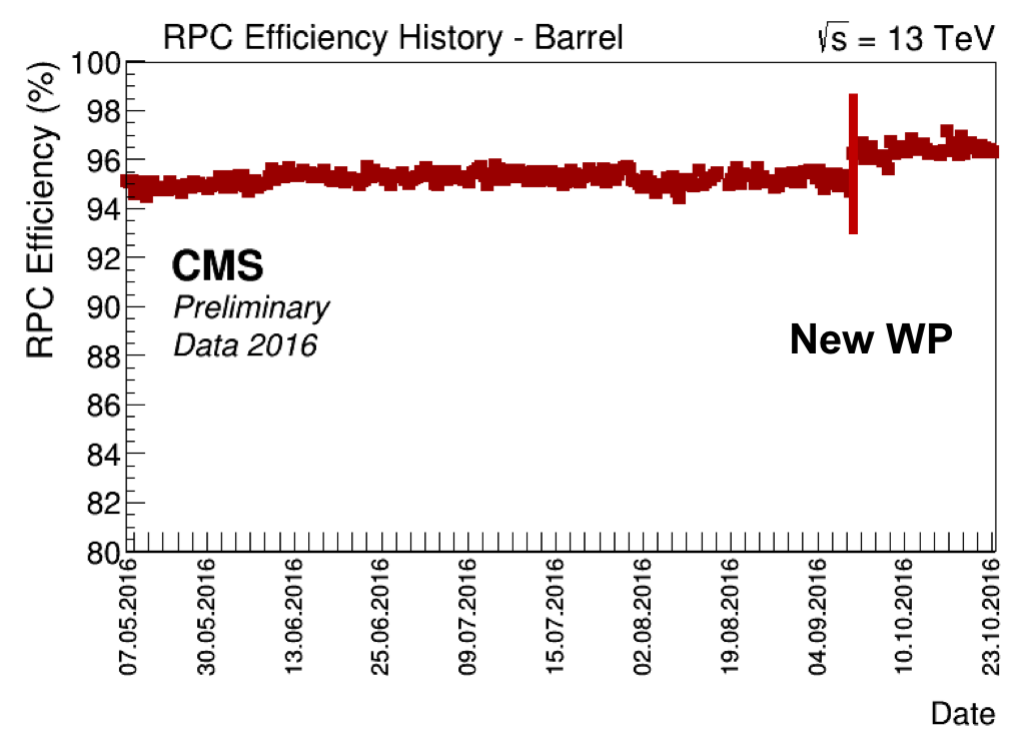

Figure 4: The plots represent the history of the efficiency for the barrel for 2016 physics data taking.

\subsection{RPC Background}

Background radiation level in the CMS muon system is one of the important factors in the overall performance. Low-momentum primary and secondary muons, low-energy gamma-rays, neutrons, and LHC beam-induced backgrounds could have an impact on performance of trigger and pattern recognition of muon tracks. In addition, excessive radiation levels can also cause premature ageing of the detectors. Main contribution in measured RPC rate is coming from background. A plot is shown in figure 5 as an example of 2016 at $13 \mathrm{TeV}$ for the fourth endcap stations.

The rate is measured directly from the link boards and was normalised to the active area of the fired strips. In the plots shown in figure 5 the $\mathrm{Y}$ axes correspond to the detector segmentation in pseudorapidity $(\eta)$, where the lowest layers $\left(R 2 \_C\right)$ are closer to the beam pipe and the outermost 


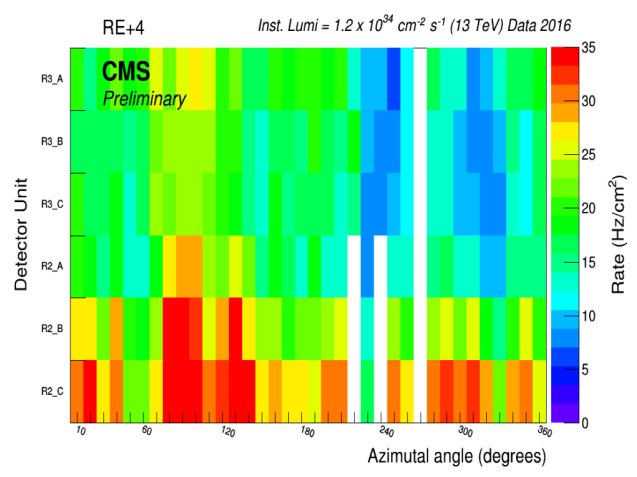

(a)

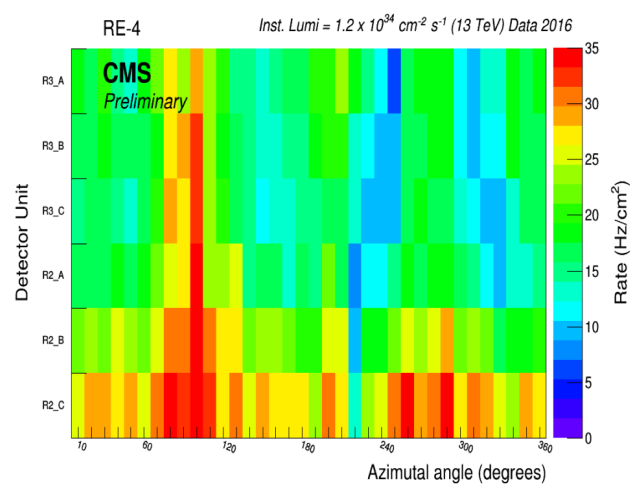

(b)

Figure 5: The detector units hit rate (in $\mathrm{Hz} / \mathrm{cm}^{2}$ ) is shown for a run at average instantaneous luminosity of $4.5 * 10^{3} 3 \mathrm{~cm}^{-2} \mathrm{~s}^{-1}$ for the newly installed RPCs in the positive (a) and negative in (b) during 2016 in at 13 $\mathrm{TeV}$.

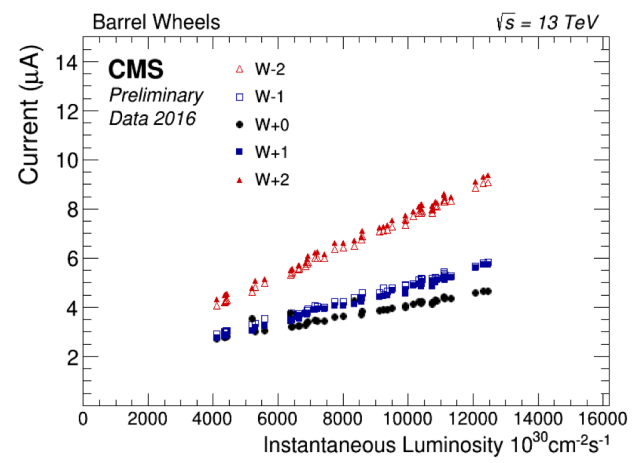

(a)

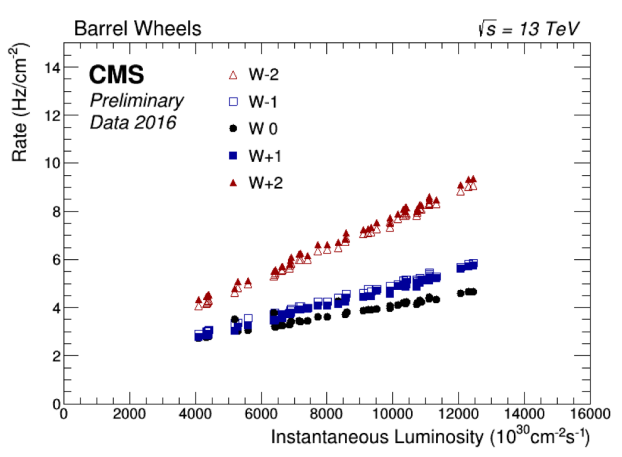

(b)

Figure 6: The plots represent the average hit rate vs. instantaneous luminosity for all barrel wheels, with $2016 p p$ collisions at $13 \mathrm{TeV}$ in (a) and currents vs. instantaneous luminosity in (b).

ones $\left(R 3 \_A\right)$ are farthest from it. As might be seen from the plots a clear $\phi$ asymmetry has been observed in both stations. The lower value in the positive one is caused by reduced background after the installation of additional layer in the rotating shielding only in the positive part. The hottest points have been spotted to be in the top vertical sectors.

As shown in figure 6, RPC rates increase approximately linearly with the luminosity of LHC. The linear behaviour can be used to extrapolate the rate for future upgrades. The rates increase for those chambers which are farther from the interaction point. 


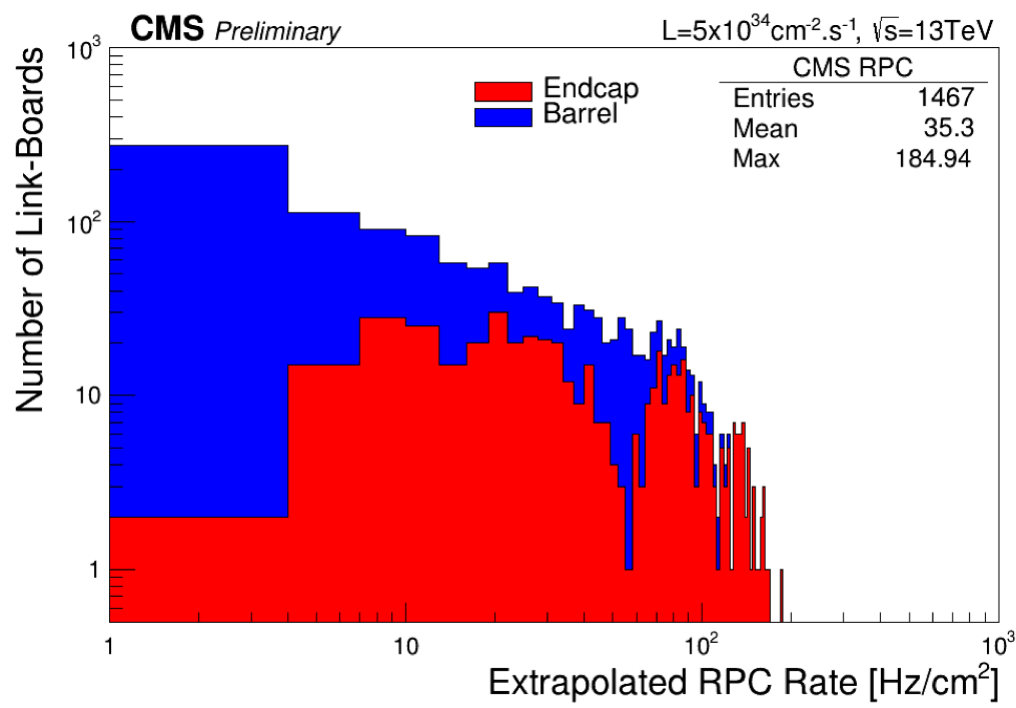

Figure 7: Extrapolation from 2016 data of single hit rate per unit area to HL-LHC conditions in the barrel and endcap

The linear dependence of the RPC hit rates on the instantaneous luminosity for every RPC chamber have been extrapolated to the instantaneous luminosity of $5 * 10^{34} \mathrm{~cm}^{-2} \mathrm{~s}^{-1}$ shown in figure7 in order to predict the expected background conditions at HL-LHC (High Luminosity LHC). The maximum expected rate is around $185 \mathrm{~Hz} / \mathrm{cm}^{2}$. The system will certified for $600 \mathrm{~Hz} / \mathrm{cm}^{2}$ (with a safety margin of 3) [9].

\section{Conclusion}

CMS RPC system was operating very well during RUN-2 (2016). After 1 year of LHC running with increasing instantaneous luminosity and 7 years from the end of RPC construction, the detector performance is within CMS specifications and stable with no degradation observed. From the measured background, no significant issues were found for running up to high luminosity scenarios.

\section{Acknowledgments}

We wish to congratulate our colleagues in the CERN accelerator departments for the excellent performance of the LHC machine. We thank the technical and administrative staff at CERN and all CMS institutes.

\section{References}

[1] L. Evans and P. Bryant, LHC Machine, J. Instrum. 3 (2008) S08001. 
[2] CMS Collaboration, The CMS experiment at the CERN LHC, J. Instrum. 3 (2008) S08004.

[3] R. Santonico and R. Cardarelli, Development of resistive plate counters, Nucl. Instrum. Meth. 187 (1981) 377.

[4] CMS Collaboration, The CMS muon project: Technical Design Report. 1997. CERN-LHCC-97-032, CMS-TDR-003.

[5] C. Camilo, The CMS Resistive Plate Chambers system-detector performance during 2011, WSPC (2012), 10.1142/9789814405072-0068.

[6] M. Abbrescia, Cosmic ray test of double-gap resistive plate chambers for the CMS experiment, Nucl. Instrum. Meth. A550 (2005) 116.

[7] CMS collaboration, The performance of the CMS muon detector in proton-proton collisions at $\sqrt{s}=7$ TeV at the LHC, J. Instrum. 8 (2013) P11002.

[8] M. A. Shah, First results of CMS RPC performance at 13 TeV, J. Instrum. 11 (2016) C12003.

[9] CMS Collaboration, Aging studies for the CMS RPC system Poster, EPS-HEP2017. 